\title{
Polarized training has greater impact on key endurance variables than threshold, high intensity, or high volume training
}

\author{
Thomas Stöggl ${ }^{1,2 *}$ and Billy Sperlich ${ }^{3}$ \\ 1 Department of Sport Science and Kinesiology, University of Salzburg, Salzburg, Austria \\ ${ }^{2}$ Department of Health Sciences, Swedish Winter Sports Research Centre, Mid Sweden University, Östersund, Sweden \\ ${ }^{3}$ Institute of Sport Science, University of Würzburg, Würzburg, Germany
}

\section{Edited by:}

Niels H. Secher, University of

Copenhagen, Denmark

Reviewed by:

Niels H. Secher, University of

Copenhagen, Denmark

Stefanos Volianitis, Aalborg

University, Denmark

*Correspondence:

Thomas Stöggl, Department of Sport Science and Kinesiology,

University of Salzburg, Schlossallee

49, 5400 Hallein/Rif, Salzburg,

Austria

e-mail: thomas.stoeggl@sbg.ac.at
Endurance athletes integrate four conditioning concepts in their training programs: high-volume training (HVT), "threshold-training" (THR), high-intensity interval training (HIIT) and a combination of these aforementioned concepts known as polarized training (POL). The purpose of this study was to explore which of these four training concepts provides the greatest response on key components of endurance performance in well-trained endurance athletes.

Methods: Forty eight runners, cyclists, triathletes, and cross-country skiers (peak oxygen uptake: $\left(\mathrm{VO}_{\text {2peak }}\right): 62.6 \pm 7.1 \mathrm{~mL} \cdot \mathrm{min}^{-1} \cdot \mathrm{kg}^{-1}$ ) were randomly assigned to one of four groups performing over 9 weeks. An incremental test, work economy and a $\mathrm{VO}_{2 \text { peak }}$ tests were performed. Training intensity was heart rate controlled.

Results: POL demonstrated the greatest increase in $\mathrm{VO}_{2 \text { peak }}\left(+6.8 \mathrm{ml} \cdot \mathrm{min} \cdot \mathrm{kg}^{-1}\right.$ or $11.7 \%$, $P<0.001)$, time to exhaustion during the ramp protocol $(+17.4 \%, P<0.001)$ and peak velocity/power $(+5.1 \%, P<0.01)$. Velocity/power at $4 \mathrm{mmol} \cdot \mathrm{L}^{-1}$ increased after $\mathrm{POL}$ $(+8.1 \%, P<0.01)$ and HIIT $(+5.6 \%, P<0.05)$. No differences in pre- to post-changes of work economy were found between the groups. Body mass was reduced by $3.7 \%$ $(P<0.001)$ following HIIT, with no changes in the other groups. With the exception of slight improvements in work economy in THR, both HVT and THR had no further effects on measured variables of endurance performance $(P>0.05)$.

Conclusion: POL resulted in the greatest improvements in most key variables of endurance performance in well-trained endurance athletes. THR or HVT did not lead to further improvements in performance related variables.

Keywords: lactate threshold, peak power, peak oxygen uptake, time to exhaustion, work economy

\section{INTRODUCTION}

Athletes participating in endurance sports such as running, cycling, and cross-country skiing integrate four conditioning concepts into their training program to maximize athletic performance. The first conditioning concept is prolonged high-volume low-intensity exercise (HVT). The second is training at or near the lactate threshold (THR); third is low-volume high-intensity interval training (HIIT) and the fourth concept is a combination of the aforementioned concepts known as "polarized" training (POL). There is a debate as to which of these training concepts may be superior in maximizing adaptations and performance.

HVT executed with low (LOW) intensity [approximately $65-75 \%$ of peak oxygen uptake $\left(\mathrm{VO}_{2 \text { peak }}\right)<80 \%$ of peak heart rate $\left(\mathrm{HR}_{\text {peak }}\right)$ or $<2 \mathrm{mmol} \cdot \mathrm{L}^{-1}$ blood lactate (Laursen and Jenkins, 2002; Seiler and Kjerland, 2006)] and prolonged duration is thought to be a fundamental training concept in preparing for endurance events. This type of exercise improves $\mathrm{VO}_{2 \text { peak }}$ by increasing stroke and plasma volume and induces molecular adaptations for capillary and mitochondrial biogenesis, thereby improving the efficiency of metabolic key components for energy fueling (Romijn et al., 1993; Midgley et al., 2006).

HIIT has revealed great improvements in athletic performance and related key variables of endurance (e.g., time to exhaustion, time trial performance, $\mathrm{VO}_{2 \text { peak }}$, maximal and submaximal running speed, running economy) in both trained and untrained individuals (Laursen and Jenkins, 2002). These improvements were largely due to increases in $\mathrm{O}_{2}$ availability, extraction and utilization and the increases in $\mathrm{VO}_{2 \text { peak }}$ (Daussin et al., 2007; Helgerud et al., 2007). A condensed 2 week block of 10-13 sessions of HIIT led to a $7 \%$ increase in $\mathrm{VO}_{2 \text { peak }}$ (Stølen et al., 2005).

Training at or close to the lactate threshold (LT) (Faude et al., 2009), referred to as "threshold training," improves endurance performance, particularly in untrained participants (Denis et al., 1984; Londeree, 1997). However, Norwegian world-class sprint cross-country skiers demonstrated greater training volume close to the LT when compared to national-level skiers (Sandbakk et al., 2011). Furthermore, in elite cross-country skiers greater 
improvements in running speed at lactate threshold and performance in a 20 -min run when exercising at an intensity eliciting 3-4 mmol. $\mathrm{L}^{-1}$ lactate compared with low intensity training $\left(<3-4 \mathrm{mmol} \cdot \mathrm{L}^{-1}\right)$ were found (Evertsen et al., 2001). In contrast, experimental and correlational data from well-trained athletes suggest that training time close to LT may be ineffective, or even counterproductive (Esteve-Lanao et al., 2007; Guellich and Seiler, 2010).

Retrospective analysis of the intensity, duration and frequency of the training load of international-level cross-country skiers (Seiler and Kjerland, 2006), rowers (Steinacker et al., 1998), cyclists (Schumacher and Mueller, 2002), and runners (Billat et al., 2001; Esteve-Lanao et al., 2005) revealed that elite endurance athletes completed most of their yearly training sessions at either intensities below ( $\sim 75 \%$ of total training volume) or well above ( $\sim 15-20 \%$ of total training volume) their LT. Six weeks of cycling using POL resulted in greater systemic adaptation in already well-trained athletes when compared to THR (Neal et al., 2013). However, no study has investigated the POL concept in well-trained endurance athletes to determine whether this concept may be superior to the aforementioned training strategies.

In many endurance sports, five key variables have been used as a benchmark to compare athletic performance in and between endurance athletes: (i) $\mathrm{VO}_{2 \text { peak }}$ (Bassett and Howley, 2000); (ii) velocity/power output at the lactate threshold $\left(\mathrm{V} / \mathrm{P}_{\mathrm{LT}}\right)$ (Bassett and Howley, 2000; Midgley et al., 2007; Faude et al., 2009); (iii) work economy (Di Prampero et al., 1986; Helgerud et al., 2001); (iv) peak running velocity or power output $\left(\mathrm{V} / \mathrm{P}_{\text {peak }}\right)$ (Midgley et al., 2007); and (v) time to exhaustion (TTE) (Laursen and Jenkins, 2002). The aim of this study was to compare the effects of four training concepts (HVT vs. THR vs. HIIT vs. POL) on the aforementioned key variables of endurance performance in welltrained athletes. We hypothesized that the POL and HIIT group would lead to superior improvements compared with HVT and THR.

\section{MATERIALS AND METHODS PARTICIPANTS}

Forty eight healthy competitive endurance athletes who participated in either cross-country skiing, cycling, triathlon, middleor long-distance running volunteered to take part in this study (mean \pm SD: age: $31 \pm 6$ years, body mass: $73.8 \pm 9 \mathrm{~kg}$, height: $180 \pm 8 \mathrm{~cm})$. All participants were well-trained [62.6 $\pm 7.1 \mathrm{~mL} \cdot \mathrm{min}^{-1} \cdot \mathrm{kg}^{-1}$ (range: $52-75 \mathrm{~mL} \cdot \mathrm{min}^{-1} \cdot \mathrm{kg}^{-1}$ )] athletes, accustomed to a workload of more than five sessions per week $\left(10-20 \mathrm{~h} \cdot \mathrm{wk}^{-1}\right)$ and had frequently been involved in endurance competitions for at least 8-20 years. Participants were members of the Austrian cross-country skiing national team $(n=8)$, running $(n=21)$, triathlon $(n=4)$ or cycling $(n=15)$ teams during or since the year before the current study. Retrospective analysis of training protocols over 6 months prior to the study revealed that none of the participants had regularly executed HIIT. All had followed a HVT training protocol with a maximum of two THR training sessions per week.

Based on the participants' baseline $\mathrm{VO}_{2 \text { peak }}$ and training mode (running or cycling), all athletes were randomized into HIIT,
HVT, THR or POL. At baseline, the four groups were not statistically different with regard to age, height, body mass or $\mathrm{VO}_{2 \text { peak }}$. During an initial visit, study details and participation requirements were explained, and written informed consent was obtained. The study received approval from the University of Salzburg Austria Ethics Committee and was conducted in accordance with the Declaration of Helsinki.

\section{DESIGN}

The intervention lasted 9 weeks plus 2 days of pre- and posttesting. All athletes ( $n=15$ cyclists; $n=3$ triathletes) engaging in cycling training trained with their own bike and completed all tests on a bicycle ergometer (Ergoline, Ergoselect 100P; Bitz, Germany) using their own cycling shoes and pedal system. Other athletes ( $n=16$ runners, $n=6$ triathletes, $n=8$ crosscountry skiers) ran during the study and completed their preand post-testing on a motorized treadmill (HP Cosmos, Saturn, Traunstein, Germany). All participants were instructed not to change their diet throughout the training period and to maintain strength training, if it was part of their training program. Participants' nutritional intake was not standardized or controlled during the study, but for the $3 \mathrm{~h}$ prior to all testing in which food intake was not permitted. The training intensity was controlled by HR based on the baseline incremental test: (i) LOW (HR at blood lactate value $<2 \mathrm{mmol} \cdot \mathrm{L}^{-1}$ ); (ii) LT (HR corresponding to a blood lactate of $3-5 \mathrm{mmol} \cdot \mathrm{L}^{-1}$ ); (iii) $\mathrm{HIGH}(>90 \%$ $H_{\mathrm{R}}$ peak $\left.)\right]$. The HR was measured during each training session and athletes documented training mode, exercise duration, and intensity in a diary. As a control and for detailed analysis, HR for all training sessions was stored digitally and analyzed retrospectively.

\section{HVT INTERVENTION}

The HVT included three blocks each lasting 3 weeks: 2 weeks of high-volume training followed by 1 week of recovery (Figure 1A). The two high volume weeks each included six training sessions with three 90 min LOW sessions, two 150-240 min LOW sessions (according to the training mode: running, cycling, or roller skiing) and one $60 \mathrm{~min}$ LT session using different types of interval training (e.g., $5 \times 7 \mathrm{~min}$ with $2 \mathrm{~min}$ recovery, $3 \times 15 \mathrm{~min}$ with 3 min recovery). The recovery week included three training sessions with two 90 min LOW sessions and one 150-180 min LOW session.

\section{THR INTERVENTION}

The THR included three blocks, each lasting 3 weeks: 2 weeks of high volume and intensity training followed by 1 week of recovery (Figure 1B). The two high volume and intensity weeks each included six training sessions with two $60 \mathrm{~min}$ interval sessions at the LT $(5 \times 6 \mathrm{~min}$ and 2 min recovery in the first block, $6 \times 7 \mathrm{~min}$ in the second block and $6 \times 8 \mathrm{~min}$ in the last block), one $90 \mathrm{~min}$ LT session with longer intervals $(3 \times 15 \mathrm{~min}$ with 3 min active recovery in the first block and $3 \times 20 \mathrm{~min}$ for the remaining two blocks), one $75 \mathrm{~min}$ session with varying changes in intensity ("fartlek") (intensities resulting in a blood lactate of $1.5-5 \mathrm{mmol} \cdot \mathrm{L}^{-1}$ ) and two $90 \mathrm{~min}$ LOW sessions. The recovery week included one $60 \mathrm{~min}$ LOW session and two $60 \mathrm{~min}$ LT interval sessions $(5 \times 6 \mathrm{~min}$ with $2 \mathrm{~min}$ of active recovery). 

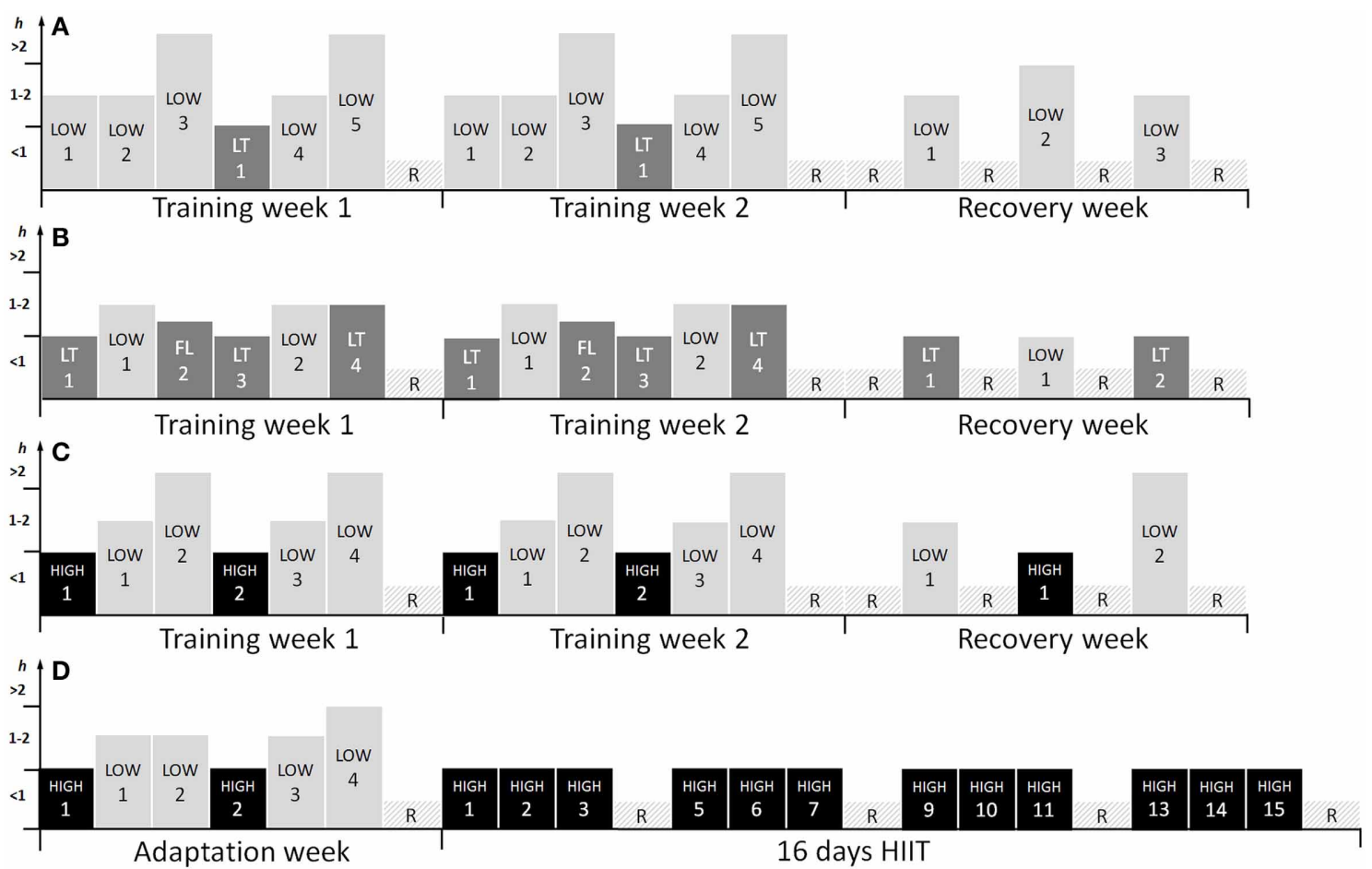

FIGURE 1 | Training program for 3-weeks of (A) high volume (HVT), (B) threshold (THR), (C) polarized (POL) training, and (D) the training program for the first block of high intensity interval training (HIIT), excluding the recovery week. LOW, low training intensity $\left(<2 \mathrm{mmol} \cdot \mathrm{L}^{-1}\right)$; $\mathrm{LT}$, training intensity around the lactate threshold $\left(3-5 \mathrm{mmol} \cdot \mathrm{L}^{-1}\right) ; \mathrm{FL}$, fartlek; HIIT, high intensity interval training ( $\left.>90 \% H R_{\text {peak }}\right)$; $R$, recovery day.

\section{HIIT INTERVENTION}

The HIIT included two interval blocks of 16 days with one adaptation week prior to and one recovery week after each block. The adaptation week included two $60 \mathrm{~min}$ HIIT sessions, three 90 min LOW sessions, one 120 min LOW session and 1 day of recovery. The condensed 16 day interval block included 12 HIIT sessions within 15 days, integrating four blocks of three HIIT sessions for three consecutive days followed by 1 day of recovery. The recovery week contained four LOW sessions of $90 \mathrm{~min}$ and 3 days without any training (not presented in Figure 1D). All of the HIIT sessions included a $20 \mathrm{~min}$ warm-up at $75 \%$ of $\mathrm{HR}_{\text {peak }}, 4 \times 4 \mathrm{~min}$ at $90-95 \%$ of $\mathrm{HR}_{\text {peak }}$ with $3 \mathrm{~min}$ active recovery and a $15 \mathrm{~min}$ cool-down at $75 \% \mathrm{HR}_{\text {peak }}$ based on the protocol proposed earlier (Helgerud et al., 2007). The LOW sessions lasted 90-150 min depending on the training mode (running vs. cycling) at an intensity resulting blood lactate of $<2 \mathrm{mmol} \cdot \mathrm{L}^{-1}$.

\section{POL INTERVENTION}

The POL included three blocks, each lasting 3 weeks: 2 weeks of high volume and intensity training followed by 1 week of recovery (Figure 1C). The high volume and intensity week included six sessions with two 60 min HIIT sessions, two 150-240 min long duration LOW sessions (duration according to training mode: cycling, running or roller skiing), which included six to eight maximal sprints of $5 \mathrm{~s}$ separated by at least $20 \mathrm{~min}$, and two $90 \mathrm{~min}$ LOW sessions. The recovery week included one $60 \mathrm{~min}$
HIIT session, one $120-180 \mathrm{~min}$ LOW session and one $90 \mathrm{~min}$ LOW session.

\section{PRE AND POST-TESTING}

All participants were asked to report well-hydrated and to refrain from consuming alcohol and caffeine for at least 24-h, as well as from engaging in strenuous exercise at least 48 -h prior to testing. The pre- and post-tests included the determination of body mass, an incremental test protocol, a work economy and $\mathrm{VO}_{2 \text { peak }}$ ramp protocol.

On the first day participants performed an incremental test on a treadmill $\left(7.2 \mathrm{~km} \cdot \mathrm{h}^{-1}\right.$; increment: $1.8 \mathrm{~km} \cdot \mathrm{h}^{-1}$ every $5 \mathrm{~min}$, with $30 \mathrm{~s}$ recovery between stages, inclination $1 \%$ ) or cycle ergometer $(80 \mathrm{~W}$; increment: $40 \mathrm{~W}$ every $5 \mathrm{~min}$, cadence $>80 \mathrm{rpm})$ until volitional exhaustion was achieved to assess the peak velocity/power output $\left(\mathrm{V} / \mathrm{P}_{\text {peak }}\right), \mathrm{HR}$, blood lactate, as well as the velocity, power output and $\mathrm{HR}$ at 2 and $4 \mathrm{mmol} \cdot \mathrm{L}^{-1}$ blood lactate $\left(\mathrm{V} / \mathrm{P}_{2}, \mathrm{~V} / \mathrm{P}_{4}\right.$ and $\left.\mathrm{HR}_{2}, \mathrm{HR}_{4}\right)$. The participants' $\mathrm{HR}$ was recorded by telemetry (Suunto t6, Helsinki, Finland) at 2-s intervals. The mean HR over the last $30 \mathrm{~s}$ of each increment was used for statistical analysis. A $20 \mu \mathrm{l}$ blood sample from the right earlobe was collected immediately after each increment, as well as 3 and $5 \mathrm{~min}$ after the completion of the test into a capillary tube (Eppendorf AG, Hamburg, Germany). All samples were analyzed amperometric-enzymatically (Biosen 5140, EKF-diagnostic $\mathrm{GmbH}$, Magdeburg, Germany) in duplicate, and the mean of the two measures was used for statistical analysis. The lactate sensor 
was calibrated before each test using a lactate standard sample of $12 \mathrm{mmol} \cdot \mathrm{L}^{-1}$. Results within a range of $\pm 0.1 \mathrm{mmol} \cdot \mathrm{L}^{-1}$ were accepted.

One day after the incremental tests, all athletes completed a combined work economy and $\mathrm{VO}_{2 \text { peak }}$ ramp protocol to determine their submaximal and peak $\mathrm{VO}_{2}\left(\mathrm{VO}_{2 \text { submax }}\right.$ and $\left.\mathrm{VO}_{2 \text { peak }}\right)$ and $\mathrm{HR}\left(\mathrm{HR}_{\text {submax }}\right.$ and $\left.\mathrm{HR}_{\text {peak }}\right)$, as well as time to exhaustion (TTE). First, the intensity for running was set at $8 \mathrm{~km} \cdot \mathrm{h}^{-1}$ (inclination: $5 \%$ ) on the treadmill, and for cycling at $200 \mathrm{~W}$ with a cadence of $>80 \mathrm{rpm}$ for $10 \mathrm{~min}$ to determine $\mathrm{VO}_{2 \text { submax }}$ and $\mathrm{HR}_{\text {submax }}$ for this intensity. The mean $\mathrm{VO}_{2}$ and $\mathrm{HR}$ during the last $5 \mathrm{~min}$ of these tests were used for statistical purposes. The intensity was then increased every $30 \mathrm{~s}$ by $0.5 \mathrm{~km} \cdot \mathrm{h}^{-1}$ (inclination: $10 \%$ ) on the treadmill or $15 \mathrm{~W}$ on the cycle ergometer until exhaustion. The overall time for the ramp test was defined as time to exhaustion (TTE). $\mathrm{VO}_{2}$ was measured with an open circuit breath-by-breath spirograph (nSpire, Zan 600 USB, Oberthulba, Germany), which was calibrated prior to each test using high precision gas ( $15.8 \% \mathrm{O}_{2}, 5 \% \mathrm{O}_{2}$ in N; Praxair, Düsseldorf, Germany) and a $1 \mathrm{~L}$ syringe (nSpire, Oberthulba, Germany). All respiratory data were averaged every $30 \mathrm{~s}$. $\mathrm{VO}_{2 \text { peak }}$ was achieved if three of the four following criteria were met: (1) plateau in $\mathrm{VO}_{2}$, i.e., an increase $<1.0 \mathrm{~mL} \cdot \mathrm{min}^{-1} \cdot \mathrm{kg}^{-1}$ despite an increase in velocity or power output; (2) respiratory exchange ratio $>1.1$; (3) $\mathrm{HR} \pm 5 \%$ of age predicted $\mathrm{HR}_{\text {peak }}$; and (4) peak blood lactate $\left(\mathrm{LA}_{\text {peak }}\right)>$ $6 \mathrm{mmol} \cdot \mathrm{L}^{-1}$ after exercise. Reliability analysis of The $\mathrm{VO}_{2 \text { peak }}$ test $(n=18)$ revealed ICC values of 0.96 for $\mathrm{VO}_{2 \text { peak }}$ and 0.98 for TTE.

\section{STATISTICAL ANALYSES}

All data exhibited a Gaussian distribution verified by the ShapiroWilk's test and, accordingly, the values are presented as means \pm $S D$. Two-Way $2 \times 4$ repeated-measures ANOVA (2 times: prepost, 4 groups) to test for global differences between pre- and post-intervention, the four training groups and the interaction effect between both factors was applied. When a significant main effect over time was observed, paired $t$-tests within each group were conducted. Based on the different units of peak power/velocity and power/velocity at 2 and $4 \mathrm{mmol} \cdot \mathrm{L}^{-1}$ blood lactate in the incremental and $\mathrm{VO}_{2 \text { peak }}$ test, percent changes between pre- to post-values were calculated, and a One-Way ANOVA between groups was performed using Tukey's post-hoc analysis. Furthermore, within group changes for these variables were calculated using Wilcoxon tests. An alpha value of $<0.05$ was considered significant. The Statistical Package for the Social Sciences (Version 20.0; SPSS Inc., Chicago, IL, USA) and Office Excel 2010 (Microsoft Corporation, Redmond, WA, USA) were used for statistical analysis.

\section{RESULTS}

Forty-one participants completed the 9 week training protocol, fulfilling more than $95 \%$ of the training program and staying within the given HR zones. Seven subjects ( 2 in HIIT, 1 in HVT and 4 in THR) withdrew from the study due to illness $(n=2)$, or were excluded due to changes in competition schedule $(n=3)$ or for not fulfilling the training protocol $(n=2)$. The total training hours, number of training sessions and their percent distribution within LOW, LTP, and HIIT are presented in Table 1. POL and HVT had higher training volume compared with THR and HIIT $(P<0.05-0.001)$, while having a similar number of training sessions $(P>0.05)$. HVT demonstrated the greatest amount of LOW, THR of LT, and HIIT in HIGH training sessions (all, $P<0.05)$.

Body mass after HIIT was reduced by $3.7 \pm 3.0 \%$ (baseline: $73.5 \pm 6.8 \mathrm{~kg}$, post: $70.7 \pm 6.5 \mathrm{~kg}, P<0.01)$ with no significant change in the HVT, THR or POL groups. The reduction in body mass after HIIT was greater compared to other training interventions $(P<0.001)$.

Percent changes in variables from pre- to post-training and between the training concepts during the $\mathrm{VO}_{2 \text { peak }}{ }^{-}$ ramp, work economy, and incremental tests are presented in Table 2. POL demonstrated the greatest increase in $\mathrm{VO}_{2 \text { peak }}$

Table 1 | The distribution of volume and training intensity within the 9 weeks training intervention (excluding strength training).

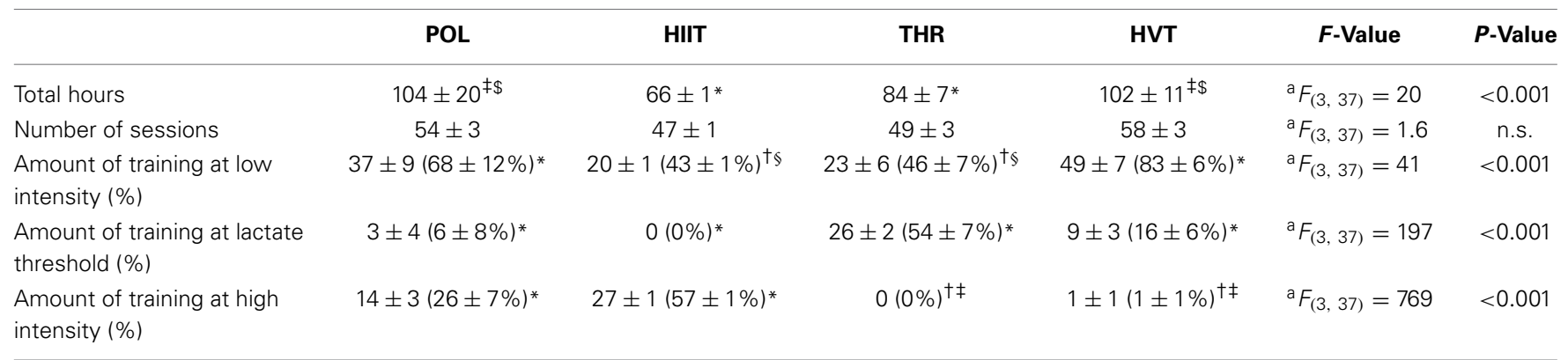

The values presented are means \pm SD. F- and P-values were obtained by One-Way ANOVA (4 training groups). POL, polarized training group; HIIT, High intensity interval training group; THR, threshold training group; HVT, high volume training group.

*Different from all other groups.

${ }^{+}$Different from training group "POL."

"Different from training group "HIIT."

\$Different from training group "THR."

`Different from training group "HVT."

${ }^{a}$ Main effect between groups.n.s., not significant. 


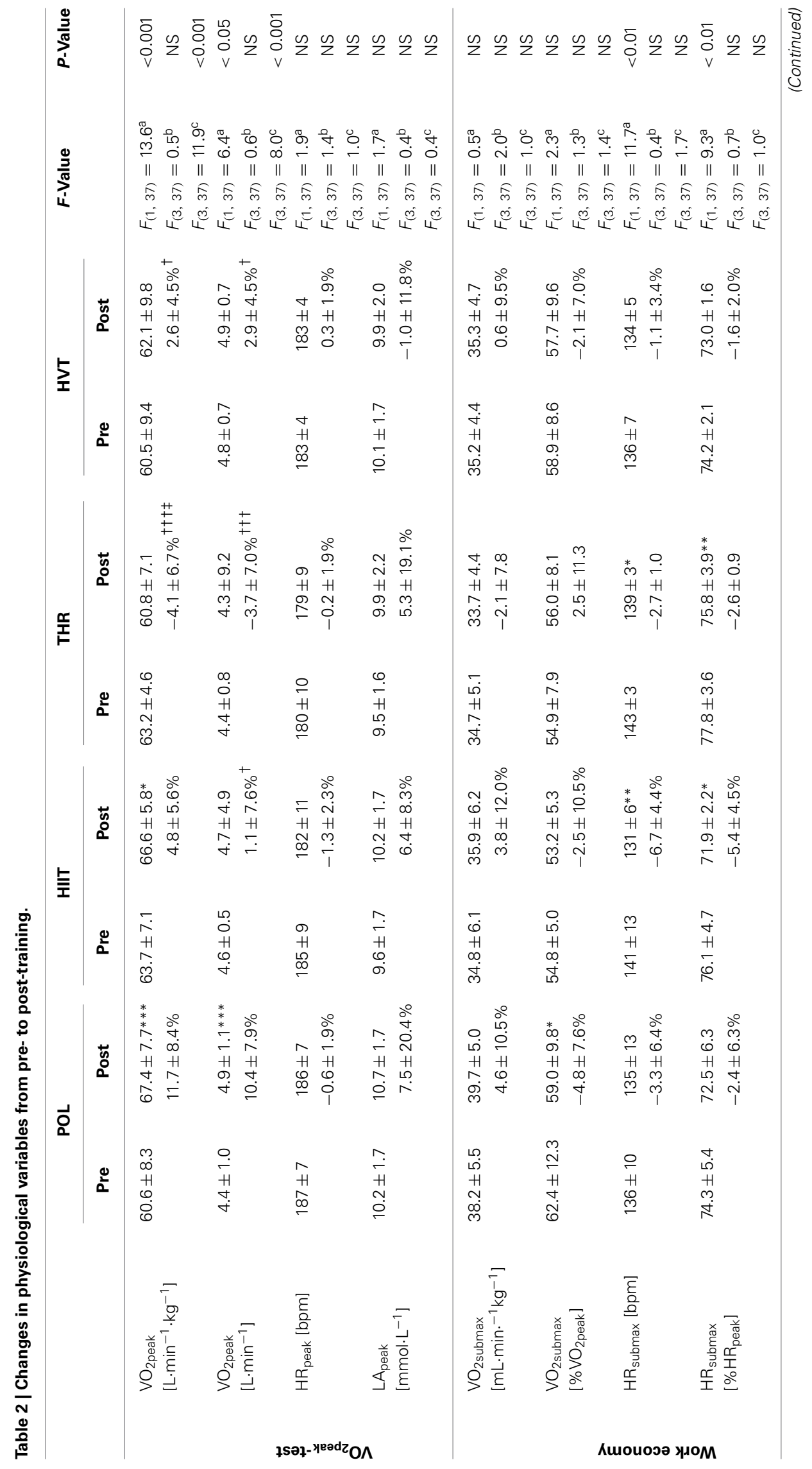




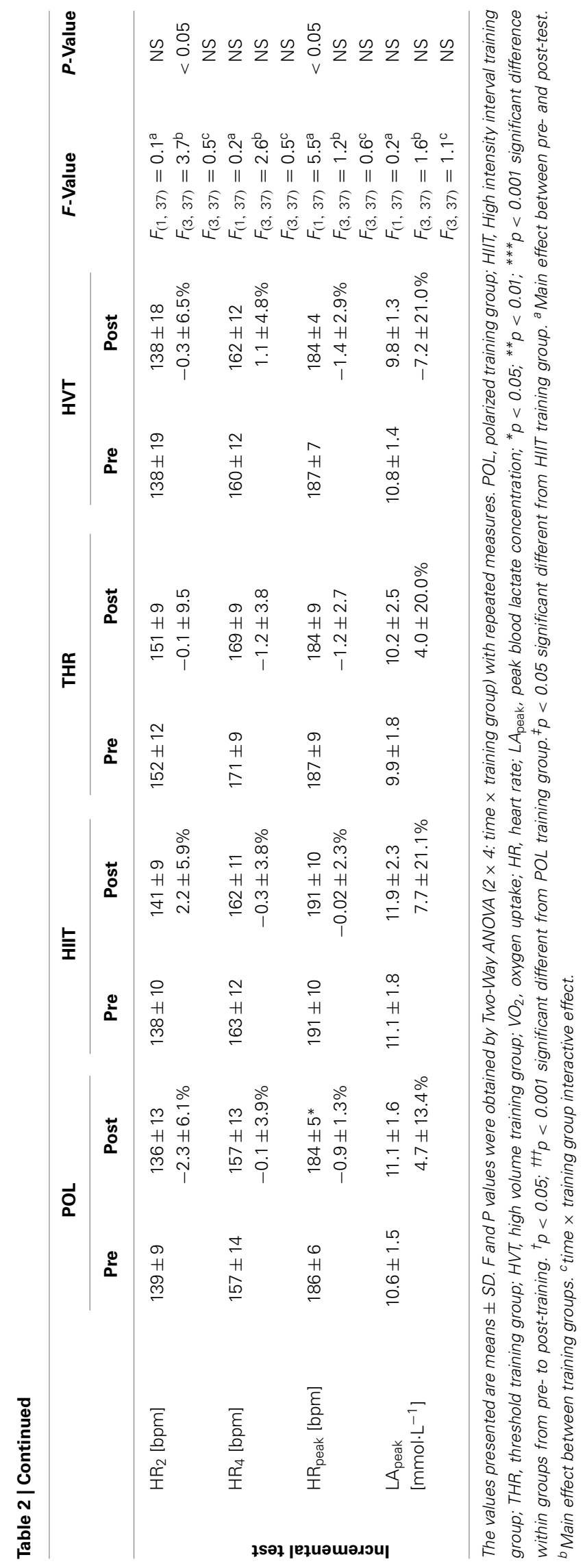

with an $11.7 \pm 8.4 \%, \quad\left(60.6 \pm 8.3-67.4 \pm 7.7 \mathrm{ml} \cdot \mathrm{min}^{-1} \cdot \mathrm{kg}^{-1}\right.$; $P<0.001)$, followed by HIIT with a $4.8 \pm 5.6 \%$ increase $(P<$ $0.05)$. The change in $\mathrm{VO}_{2 \text { peak }}$ in $\mathrm{POL}$ was higher compared to THR and HVT $(P<0.001$ and $P<0.05)$. Absolute $\mathrm{VO}_{2 \text { peak }}$ increased in POL by $10.4 \pm 7.9 \%(P<0.001)$, which was greater compared with the other training concepts (HIIT and HVT $P<$ 0.05 , THR $P<0.001)$. No changes from pre to post and no differences between training groups with respect to $\mathrm{HR}_{\text {peak }}, \mathrm{LA}_{\text {peak }}$, and $\mathrm{HR}_{2} \& 4$ were detected $(P>0.05)$. Work economy increased following HIIT $(-6.7 \pm 4.4 \%$ decrease in HR, $P<0.01)$ and THR $(-2.7 \pm 1.0 \%$ decrease in $\mathrm{HR}, P<0.05)$ with no significant differences between the other concepts. Work economy expressed as percent of $\mathrm{VO}_{2 \text { peak }}$ was only improved after POL $(-4.8 \pm 7.6 \%, P<0.05)$ with no significant differences between the other training groups.

The changes in TTE, $\mathrm{V} / \mathrm{P}_{\text {peak }}$ and $\mathrm{V} / \mathrm{P}_{2} \& 4$ from pre- to posttraining and between the single training groups are presented in Table 3. The largest percentage increase in TTE, assessed using the $\mathrm{VO}_{2 \text { peak }}$ ramp test, was observed in response to POL $(+17.4 \%$, $P<0.001)$ followed by HIIT $(+8.8 \%, P<0.01)$; however, no statistical differences were found between the four training concepts. $\mathrm{V} / \mathrm{P}_{\text {peak }}$ in the incremental test increased in response to POL and HIIT $(5.1 \pm 3.0 \%$ and $4.4 \pm 2.8 \%$, both $P<0.01)$ with both groups demonstrating greater changes than HVT $(P<0.01$ and $P<0.05) . \mathrm{V} / \mathrm{P}_{4}$ increased after POL $(8.1 \pm 4.6 \%, P<0.01)$ and HIIT $(5.6 \pm 4.8 \%, P<0.01)$ demonstrating greater changes after POL compared to THR and HVT (both $P<0.05$ ).

\section{DISCUSSION}

The purpose was to determine whether HIIT, HVT, THR, or POL provides the greatest impact on key variables of endurance performance in well-trained athletes. The main findings were that (1) POL led to the greatest improvement in $\mathrm{VO}_{2 \text { peak }}$ TTE and $\mathrm{V} / \mathrm{P}_{\text {peak }} ;(2) \mathrm{V} / \mathrm{P}_{4}$ increased after POL and HIIT; (3) no significant differences in work economy were observed pre to post between any of the groups; and finally (4) body mass decreased by $3.7 \%$ in response to HIIT.

There are several challenges associated with conducting an exercise training intervention such as the one presented here. Firstly, the compliance of all athletes is paramount to the successful completion of the study and for the subsequent examination of the intervention. The athletes attended more than $95 \%$ of all training sessions and all completed their predetermined training load (intensity based on HR zones, duration, and frequency), which was confirmed by logging the daily training dose in a diary and retrospective analysis of HR data. Secondly, an experimental study is difficult to conduct in elite athletes because typically neither the athletes nor their coaches like to have the athletes' training intensity, duration or frequency altered. However, we successfully managed to conduct the current study in well-trained male and female athletes $\left(\mathrm{VO}_{2 \text { peak }}: 52-75 \mathrm{~mL} \cdot \mathrm{min}^{-1} \cdot \mathrm{kg}^{-1}\right)$ over a 9 week period.

Of the four training concepts, $\mathrm{POL}$ resulted in the greatest increase in $\mathrm{VO}_{2 \text { peak }}$, TTE, $\mathrm{V} / \mathrm{P}_{\text {peak }}$ and, together with HIIT, in $\mathrm{V} / \mathrm{P}_{4}$. As mentioned, POL was confirmed by retro-perspective analysis of the intensity, duration and frequency distribution of the training load in highly trained athletes (Steinacker et al., 
Table 3 | Per cent changes in velocity (V) and power (P) and at various lactate thresholds as well as peak velocity and power.

\begin{tabular}{lcccrrr}
\hline & POL & HIIT & THR & HVT & F-Value & P-Value \\
\hline TTE & $17.4 \pm 16.1^{* * *}$ & $8.8 \pm 8.6^{* *}$ & $6.2 \pm 9.0$ & $8.0 \pm 10.3$ & ${ }^{a} F_{(3,37)}=2.0$ & NS \\
V/P & $9.3 \pm 12.4$ & $12.1 \pm 8.8^{* *}$ & $2.0 \pm 13.8$ & $0.8 \pm 13.3$ & ${ }^{a} F_{(3,37)}=1.9$ & $\mathrm{NS}$ \\
$\mathrm{V} / \mathrm{P}_{4}$ & $8.1 \pm 4.6^{* *}$ & $5.6 \pm 4.8^{*}$ & $1.4 \pm 4.3^{\dagger}$ & $1.2 \pm 6.6^{\dagger}$ & ${ }^{a} F_{(3,37)}=4.5$ & $<0.01$ \\
V/P peak & $5.1 \pm 3.0^{* *}$ & $4.4 \pm 2.8^{* *}$ & $1.8 \pm 4.8$ & $-1.5 \pm 4.9^{\dagger \dagger \neq}$ & ${ }^{a} F_{(3,37)}=4.6$ & $<0.01$ \\
\hline
\end{tabular}

The values presented are means $\pm S D$. F and $P$ values were obtained by One-Way ANOVA (4 training groups) calculated over the per cent differences between preto post-training. POL, polarized training group; HIIT, High intensity interval training group; THR, threshold training group; HVT, high volume training group; TTE, time to exhaustion during the ramp test; $V / P_{2}$, velocity or power at $2 \mathrm{mmol} \cdot \mathrm{L}^{-1} ; \mathrm{V} / \mathrm{P}_{4}$, velocity or power at $4 \mathrm{mmol} \cdot \mathrm{L}^{-1} ; \mathrm{V} / \mathrm{P}_{\text {peak, }}$, peak velocity or power in the incremental test; ${ }^{*} p<0.05 ;{ }^{*} p<0.01 ;{ }^{* *} p<0.001$ significant difference within groups from pre- to post-training.

${ }^{t} p<0.05 ;{ }^{H} p<0.01$ significant different from POL training group.

${ }^{\ddagger} p<0.05$ significant different from HIIT training group.

${ }^{a}$ Main effect between groups.

1998; Billat et al., 2001; Schumacher and Mueller, 2002; Seiler and Kjerland, 2006; Esteve-Lanao et al., 2007). In these studies, it was demonstrated that endurance athletes perform approximately $75 \%$ of their yearly training program either below or well above $(\sim 15-20 \%)$ the LT, but little at the LT. In the current study, POL mimicked this distribution (LOW $=68 \%$, LTP $=6 \%$, HIGH $=26 \%$ ). Only the study of Neal et al. (2013) demonstrated that 6 weeks of POL resulted in greater systemic adaptation in trained cyclists when compared to THR, hence supporting our findings.

In moderately trained persons, HVT improves metabolic and hemodynamic adaptations over 3 days (Green et al., 1987, 1990; Coyle, 1999). However, a greater volume of training ( $\sim 3-5$ weeks with 3-5 sessions $\cdot \mathrm{wk}^{-1}$ ) is needed to improve $\mathrm{VO}_{2 \text { peak }}$ (Laursen and Jenkins, 2002). One reason due to why athletes may choose a high amount of HVT may be due to that HVT leads to improved fat and glucose utilization (Romijn et al., 1993), which is beneficial for long lasting endurance events. Therefore, it might be reasonable to implement HVT in the training programs of elite endurance athletes for improving oxidative flux, which is important for converting energy aerobically and recovery after and during HIIT sessions with large anaerobic portions. When HVT becomes the major component of a training program and HIIT sessions are neglected, no further improvement in $\mathrm{VO}_{2 \text { peak }}$ and performance in already well-trained athletes occur (Costill et al., 1988; Laursen and Jenkins, 2002); in line with the findings of the present study. Further improvements of well-trained athletes require adding high intensity training sessions to HVT, as demonstrated in POL. However, due to that the participants of this study mainly used HVT prior to this experiment, the HVT model might not have provided an adequate stimulus for further adaptations.

The advantage of HIIT compared to HVT lies in a shorter period of training time for similar muscular adaptations (Gibala et al., 2006; Burgomaster et al., 2008). In response to HIIT, several central and peripheral adaptations including increased stroke (Helgerud et al., 2007) and blood volume (Shepley et al., 1992), $\mathrm{O}_{2}$ extraction (Daussin et al., 2007), and improvements in aerobic and anaerobic metabolism (Macdougall et al., 1998), such as increased mitochondrial biogenesis and oxidative capacity, have been reported (Gibala et al., 2006; Daussin et al., 2007, 2008; Burgomaster et al., 2008). The aforementioned adaptations in response to HIIT explain the often documented increases in TTE, time trial performance (Lindsay et al., 1996), lactate and ventilatory threshold (Acevedo and Goldfarb, 1989; Edge et al., 2005) and $\mathrm{VO}_{2 \text { peak }}$ (Laursen and Jenkins, 2002; Gibala et al., 2006; Midgley et al., 2006; Daussin et al., 2007, 2008; Burgomaster et al., 2008).

The present study, as well as that of Helgerud et al. (2007), demonstrated that training at or near $\mathrm{VO}_{2 \text { peak }}$ may be more effective in enhancing $\mathrm{VO}_{2 \text { peak }}$ when compared to HVT or THR. However, POL, a combination of HVT and HIIT, may be superior for enhancing $\mathrm{VO}_{2 \text { peak }}$ and performance. Numerous studies using "blocked" or "condensed" HIIT (i.e., several HIIT session in 1 or 2 weeks) aim to increase $\mathrm{VO}_{2 \text { peak }}$ (Stølen et al., 2005). Furthermore, in HIIT intervention studies (2-3 HIIT sessions per week), $\mathrm{VO}_{2 \text { peak }}$ increased approximately $9 \%$ over a 10 week training intervention (McMillan et al., 2005) and $11 \%$ over a 6 week training intervention (Helgerud et al., 2001) in youth and junior soccer players, suggesting a $0.5 \%$ increase in $\mathrm{VO}_{2 \text { peak }}$ per HIIT training session. In the current study, the increase in $\mathrm{VO}_{2 \text { peak }}$ following 9 weeks of HIIT (27 HIIT sessions) was 4.8\% (0.18\% increase per training session), while POL resulted in an $11.7 \%$ increase in $\mathrm{VO}_{2 \text { peak }}$ with fewer HIIT sessions (14 HIIT) $(0.84 \%$ increase per training session). This result may be explained by: (1) peak adaptation might have been reached following the first HIIT block, and therefore, repeated HIIT bouts did not produce any further improvements in $\mathrm{VO}_{2 \text { peak }}$ or performance, or (2) the combination of HVT and HIIT, much like in POL, leads to greater long-term adaptations in endurance performance than with exclusively HIIT or HVT.

THR improves $\mathrm{VO}_{2 \text { peak }}$, lactate or ventilatory thresholds and endurance performance in untrained persons (Denis et al., 1984; Londeree, 1997; Gaskill et al., 2001). These findings contrast those of the current study, as we did not observe improvements in $\mathrm{VO}_{2 \text { peak }}, \mathrm{V} / \mathrm{P}_{4}, \mathrm{TTE}$ or $\mathrm{V} / \mathrm{P}_{\text {peak }}$ in our elite athletes in response to THR. Additionally, it is possible that in well-trained endurance athletes, repeated training bouts at LT might generate unwarranted sympathetic stress (Chwalbinska-Moneta et al., 1998), while offering no further stimulus for performance enhancement (Londeree, 1997). In this context, especially within the THR group, significant variability in the individual changes in $\mathrm{VO}_{2 \text { peak }}$ from pre- to post-intervention were observed (range: -20 to 
$+4 \%$ ). However, some THR training might be beneficial to well-trained athletes since world-class sprint cross-country skiers demonstrated greater training volume in the low and moderate intensity zones compared with national-level skiers (Sandbakk et al., 2011).

The body mass of the well-trained athletes decreased by $3.7 \%$ (approximately $3 \mathrm{~kg}$ ) after HIIT, but not in response to the other training concepts. HIIT favors lipid oxidation and promotes adipose tissue loss (Perry et al., 2008; Boutcher, 2011). Depending on the athlete's baseline value, reduction in body mass may negatively impact immune function and overall health, as well as induce a catabolic state. Training blocks with increased volume and/or exercise intensity might induce symptoms of "overreaching," reduced physical capacity, burnout symptoms including tiredness, and lack of energy (Angeli et al., 2004). However, despite the large differences in the individual responses in some of the training groups, none of the athletes demonstrated reduced TTE or $\mathrm{V} / \mathrm{P}_{\text {peak }}$ after the study, nor did they report any of the aforementioned symptoms during and after the 9 week intervention. Based on the observed changes in body mass and smaller increases in $\mathrm{VO}_{2 \text { peak }}$ in the HIIT group compared with previously published data (McMillan et al., 2005; Stølen et al., 2005; Helgerud et al., 2007), longer blocks of training periods with high intensities could provoke these symptoms.

Except for significant decreases in $\% \mathrm{VO}_{2}$ peak in the POL group and $\mathrm{HR}_{\text {submax }} / \% \mathrm{HR}_{\text {peak }}$ in the HIIT and THR groups (with no group differences), no improvements in work economy were found in the current study. Helgerud et al. (2007) reported a 5\% improvement in running economy after THR, HIIT, and HVT with no differences between groups. These improvements were mainly attributed to an increased amount of running training. Therefore, the applied training regimes were largely responsible for the changes in $\mathrm{V} / \mathrm{P}_{\text {peak }}$ and $\mathrm{VO}_{2 \text { peak }}$, while work economy remained fairly constant. $\mathrm{V} / \mathrm{P}_{4}$ was only improved in POL $(8.1 \%)$ and HIIT (5.6\%). This is consistent with findings demonstrating that running velocity at lactate threshold follows the improvements in $\mathrm{VO}_{2 \text { peak }}$ (Helgerud et al., 2001; McMillan et al., 2005).

\section{LIMITATIONS AND PERSPECTIVES}

Standardized methodology of performance diagnostics (incremental test and $\mathrm{VO}_{2 \text { peak }}$ ramp protocol) was utilized to evaluate the effects of the four endurance training interventions on key variables of endurance performance. However, a direct transfer to specific competition situation (e.g., time trial) need to be established in future research. Furthermore, the increase of about $11 \%$ in $\mathrm{VO}_{2 \text { peak }}$ with POL within 9 weeks is large for well-trained endurance and has to be put in perspective within the annual training periodization. Long-term training studies are warranted to evaluate these aspects.

\section{CONCLUSION}

In this study of elite athletes performing HIIT, HVT, THR or POL training, POL results in the greatest improvements in key variables of endurance performance $\left(\mathrm{VO}_{2 \text { peak }}, \mathrm{TTE}, \mathrm{V} / \mathrm{P}_{\text {peak }}\right.$, and $\left.\mathrm{V} / \mathrm{P}_{4}\right)$. HIIT led to a decrease in body mass and less pronounced increases in $\mathrm{VO}_{2 \text { peak }}$ compared with previous findings using short term (1-2 weeks) HIIT, suggesting that a 9 week HIIT should be applied with care. Exclusive training with THR or HVT did not lead to further improvements in endurance performance related variables in well-trained athletes.

\section{DISCLOSURE OF FUNDING}

No funding was received for this work from the National Institutes of Health, the Welcome Trust, the Howard Hughes Medical Institute, or other funding agencies to PubMed Central. None of the authors had any professional relationships with companies or manufacturers who will benefit from the results of the present study. The authors declare no conflict of interest.

\section{AUTHOR CONTRIBUTIONS}

Conception and design of the experiments: Thomas Stöggl, Billy Sperlich. Performance of the experiments: Thomas Stöggl. Data analysis: Thomas Stöggl, Billy Sperlich. Preparation of the manuscript: Thomas Stöggl and Billy Sperlich. Both authors read and approved the final manuscript.

\section{ACKNOWLEDGMENTS}

The authors would like to thank the athletes, coaches, and research assistants involved in this study for their participation, enthusiasm, and cooperation. The authors would like to express appreciation for the support of Donna Kennedy. Special thanks to Julia Stöggl, Andreas Hochwimmer and Thomas Damisch for their great assistance in recruitment, care and control of the athletes during the training intervention and pre- and post-testing.

\section{REFERENCES}

Acevedo, E. O., and Goldfarb, A. H. (1989). Increased training intensity effects on plasma lactate, ventilatory threshold, and endurance. Med. Sci. Sports Exerc. 21, 563-568. doi: 10.1249/00005768-198910000-00011

Angeli, A., Minetto, M., Dovio, A., and Paccotti, P. (2004). The overtraining syndrome in athletes: a stress-related disorder. J. Endocrinol. Invest. 27, 603-612.

Bassett, D. R. Jr., and Howley, E. T. (2000). Limiting factors for maximum oxygen uptake and determinants of endurance performance. Med. Sci. Sports Exerc. 32, 70-84. doi: 10.1097/00005768-200001000-00012

Billat, V. L., Demarle, A., Slawinski, J., Paiva, M., and Koralsztein, J. P. (2001). Physical and training characteristics of top-class marathon runners. Med. Sci. Sports Exerc. 33, 2089-2097. doi: 10.1097/00005768-200112000-00018

Boutcher, S. H. (2011). High-intensity intermittent exercise and fat loss. J. Obes. 2011, 868305. doi: 10.1155/2011/868305

Burgomaster, K. A., Howarth, K. R., Phillips, S. M., Rakobowchuk, M., Macdonald, M. J., McGee, S. L., et al. (2008). Similar metabolic adaptations during exercise after low volume sprint interval and traditional endurance training in humans. J. Physiol. 586, 151-160. doi: 10.1113/jphysiol.2007.142109

Chwalbinska-Moneta, J., Kaciuba-Uscilko, H., Krysztofiak, H., Ziemba, A., Krzeminski, K., Kruk, B., et al. (1998). Relationship between EMG, blood lactate, and plasma catecholamine tresholds during graded exercise in men. J. Physiol. Phyrmacol. 49, 433-441.

Costill, D. L., Flynn, M. G., Kirwan, J. P., Houmard, J. A., Mitchell, J. B., Thomas, R., et al. (1988). Effects of repeated days of intensified training on muscle glycogen and swimming performance. Med. Sci. Sports Exerc. 20, 249-254. doi: 10.1249/00005768-198806000-00006

Coyle, E. F. (1999). Physiological determinants of endurance exercise performance. J. Sci. Med. Sport 2, 181-189. doi: 10.1016/S1440-2440(99)80172-8

Daussin, F. N., Ponsot, E., Dufour, S. P., Lonsdorfer-Wolf, E., Doutreleau, S., Geny, B., et al. (2007). Improvement of VO2max by cardiac output and oxygen extraction adaptation during intermittent versus continuous endurance training. Eur. J. Appl. Physiol. 101, 377-383. doi: 10.1007/s00421-007-0499-3

Daussin, F. N., Zoll, J., Ponsot, E., Dufour, S. P., Doutreleau, S., Lonsdorfer, E., et al. (2008). Training at high exercise intensity promotes qualitative adaptations of mitochondrial function in human skeletal muscle. J. Appl. Physiol. 104, 1436-1441. doi: 10.1152/japplphysiol.01135.2007 
Denis, C., Dormois, D., and Lacour, J. R. (1984). Endurance training, VO2 max, and OBLA: a longitudinal study of two different age groups. Int. J. Sports Med. 5, 167-173. doi: 10.1055/s-2008-1025899

Di Prampero, P. E., Atchou, G., Bruckner, J. C., and Moia, C. (1986). The energetics of endurance running. Eur. J. Appl. Physiol. Occup. Physiol. 55, 259-266. doi: 10.1007/BF02343797

Edge, J., Bishop, D., Goodman, C., and Dawson, B. (2005). Effects of high- and moderate-intensity training on metabolism and repeated sprints. Med. Sci. Sports Exerc. 37, 1975-1982. doi: 10.1249/01.mss.0000175855.35403.4c

Esteve-Lanao, J., Foster, C., Seiler, S., and Lucia, A. (2007). Impact of training intensity distribution on performance in endurance athletes. J. Strength Cond. Res. 21 , 943-949. doi: 10.1519/00124278-200708000-00048

Esteve-Lanao, J., San Juan, A. F., Earnest, C. P., Foster, C., and Lucia, A. (2005). How do endurance runners actually train? Relationship with competition performance. Med. Sci. Sports Exerc. 37, 496-504. doi: 10.1249/01.MSS.0000155393.78744.86

Evertsen, F., Medbo, J. I., and Bonen, A. (2001). Effect of training intensity on muscle lactate transporters and lactate threshold of cross-country skiers. Acto Physiol. Scand. 173, 195-205. doi: 10.1046/j.1365-201X.2001.00871.x

Faude, O., Kindermann, W., and Meyer, T. (2009). Lactate threshold concepts: how valid are they? Sports Med. 39, 469-490. doi: 10.2165/00007256-20093906000003

Gaskill, S. E., Walker, A. J., Serfass, R. A., Bouchard, C., Gagnon, J., Rao, D. C., et al. (2001). Changes in ventilatory threshold with exercise training in a sedentary population: the HERITAGE family study. Int. J. Sports Med. 22, 586-592. doi: 10.1055/s-2001-18522

Gibala, M. J., Little, J. P., van Essen, M., Wilkin, G. P., Burgomaster, K. A., Safdar, A., et al. (2006). Short-term sprint interval versus traditional endurance training: similar initial adaptations in human skeletal muscle and exercise performance. J. Physiol. 575, 901-911. doi: 10.1113/jphysiol.2006.112094

Green, H. J., Jones, L. L., Hughson, R. L., Painter, D. C., and Farrance, B. W. (1987). Training-induced hypervolemia: lack of an effect on oxygen utilization during exercise. Med. Sci. Sports Exerc. 19, 202-206. doi: 10.1249/00005768-19870600000003

Green, H. J., Jones, L. L., and Painter, D. C. (1990). Effects of short-term training on cardiac function during prolonged exercise. Med. Sci. Sports Exerc. 22, 488-493. doi: 10.1249/00005768-199008000-00012

Guellich, A., and Seiler, S. (2010). Lactate profile changes in relation to training characteristics in junior elite cyclists. Int. J. Sports Physiol. Perform. 5, 316-327.

Helgerud, J., Engen, L. C., Wisloff, U., and Hoff, J. (2001). Aerobic endurance training improves soccer performance. Med. Sci. Sports Exerc. 33, 1925-1931. doi: 10.1097/00005768-200111000-00019

Helgerud, J., Hoydal, K., Wang, E., Karlsen, T., Berg, P., Bjerkaas, M., et al. (2007). Aerobic high-intensity intervals improve VO2max more than moderate training. Med. Sci. Sports Exerc. 39, 665-671. doi: 10.1249/mss.0b013e31803 04570

Laursen, P. B., and Jenkins, D. G. (2002). The scientific basis for high-intensity interval training: optimising training programmes and maximising performance in highly trained endurance athletes. Sports Med. 32, 53-73. doi: 10.2165/00007256-200232010-00003

Lindsay, F. H., Hawley, J. A., Myburgh, K. H., Schomer, H. H., Noakes, T. D. and Dennis, S. C. (1996). Improved athletic performance in highly trained cyclists after interval training. Med. Sci. Sports Exerc. 28, 1427-1434. doi: 10.1097/00005768-199611000-00013

Londeree, B. R. (1997). Effect of training on lactate/ventilatory thresholds: a metaanalysis. Med. Sci. Sports Exerc. 29, 837-843. doi: 10.1097/00005768-19970600000016

Macdougall, J. D., Hicks, A. L., Macdonald, J. R., McKelvie, R. S., Green, H. J., and Smith, K. M. (1998). Muscle performance and enzymatic adaptations to sprint interval training. J. Appl. Physiol. 84, 2138-2142.
McMillan, K., Helgerud, J., Grant, S. J., Newell, J., Wilson, J., Macdonald, R., et al. (2005). Lactate threshold responses to a season of professional British youth soccer. Br. J. Sports Med. 39, 432-436. doi: 10.1136/bjsm.2004.012260

Midgley, A. W., McNaughton, L. R., and Jones, A. M. (2007). Training to enhance the physiological determinants of long-distance running performance: can valid recommendations be given to runners and coaches based on current scientific knowledge? Sports Med. 37, 857-880. doi: 10.2165/00007256-2007 37100-00003

Midgley, A. W., McNaughton, L. R., and Wilkinson, M. (2006). Is there an optimal training intensity for enhancing the maximal oxygen uptake of distance runners?: empirical research findings, current opinions, physiological rationale and practical recommendations. Sports Med. 36, 117-132. doi: 10.2165/00007256200636020-00003

Neal, C. M., Hunter, A. M., Brennan, L., O’Sullivan, A., Hamilton, D. L., De Vito, G., et al. (2013). Six weeks of a polarized training-intensity distribution leads to greater physiological and performance adaptations than a threshold model in trained cyclists. J. Appl. Physiol. 114, 461-471. doi: 10.1152/japplphysiol.00652.2012

Perry, C. G., Heigenhauser, G. J., Bonen, A., and Spriet, L. L. (2008). High-intensity aerobic interval training increases fat and carbohydrate metabolic capacities in human skeletal muscle. Appl. Physiol. Nutr. Metab. 33, 1112-1123. doi: 10.1139/H08-097

Romijn, J. A., Coyle, E. F., Sidossis, L. S., Gastaldelli, A., Horowitz, J. F., Endert, E., et al. (1993). Regulation of endogenous fat and carbohydrate metabolism in relation to exercise intensity and duration. Am. J. Physiol. 265, E380-E391.

Sandbakk, O., Holmberg, H. C., Leirdal, S., and Ettema, G. (2011). The physiology of world-class sprint skiers. Scand. J. Med. Sci. Sports 21, e9-e16. doi: 10.1111/j.1600-0838.2010.01117.x

Schumacher, Y. O., and Mueller, P. (2002). The 4000-m team pursuit cycling world record: theoretical and practical aspects. Med. Sci. Sports Exerc. 34, 1029-1036. doi: 10.1097/00005768-200206000-00020

Seiler, K. S., and Kjerland, G. O. (2006). Quantifying training intensity distribution in elite endurance athletes: is there evidence for an "optimal" distribution? Scand. J. Med. Sci. Sports 16, 49-56. doi: 10.1111/j.1600-0838.2004.00418.x

Shepley, B., Macdougall, J. D., Cipriano, N., Sutton, J. R., Tarnopolsky, M. A., and Coates, G. (1992). Physiological effects of tapering in highly trained athletes. J. Appl. Physiol. 72, 706-711.

Steinacker, J. M., Lormes, W., Lehmann, M., and Altenburg, D. (1998). Training of rowers before world championships. Med. Sci. Sports Exerc. 30, 1158-1163. doi: 10.1097/00005768-199807000-00022

Stølen, T., Chamari, K., Castagna, C., and Wisloff, U. (2005). Physiology of soccer: an update. Sports Med. 35, 501-536. doi: 10.2165/00007256-200535060-00004

Conflict of Interest Statement: The authors declare that the research was conducted in the absence of any commercial or financial relationships that could be construed as a potential conflict of interest.

Received: 02 October 2013; accepted: 16 January 2014; published online: 04 February 2014

Citation: Stöggl T and Sperlich B (2014) Polarized training has greater impact on key endurance variables than threshold, high intensity, or high volume training. Front Physiol. 5:33. doi: 10.3389/fphys.2014.00033

This article was submitted to Exercise Physiology, a section of the journal Frontiers in Physiology.

Copyright (C) 2014 Stöggl and Sperlich. This is an open-access article distributed under the terms of the Creative Commons Attribution License (CC BY). The use, distribution or reproduction in other forums is permitted, provided the original author(s) or licensor are credited and that the original publication in this journal is cited, in accordance with accepted academic practice. No use, distribution or reproduction is permitted which does not comply with these terms. 